\title{
Inactivation of Clostridioides Difficile Spores in Carpeting and Upholstery to Reduce Disease Recurrence in Households and Nursing Care Facilities
}

Michael J Sadowsky ${ }^{1,2,3^{*}}$, Raymond Erickson ${ }^{1}$, Ping Wang ${ }^{1}$, Matthew J Hamilton ${ }^{1}$, Melissa Hoo ${ }^{1}$, Thomas Kaiser ${ }^{1}$, Kathryn Fryxell, ${ }^{1}$, and Alexander Khoruts ${ }^{1,4^{* *}}$

${ }^{1}$ BioTechnology Institute, University of Minnesota, St. Paul, MN, United States.

${ }^{2}$ Department of Soil, Water and Climate, University of Minnesota, St. Paul, MN, United States.

${ }^{3}$ Department of Plant and Microbial Biology, University of Minnesota, St. Paul, MN, United States.

${ }^{4}$ Department of Medicine, Division of Gastroenterology, Hepatology and Nutrition, University of Minnesota, Minneapolis, MN, United States.

\section{Article Details \\ Article Type: Research Article \\ Received date: $28^{\text {th }}$ October, 2021 \\ Accepted date: $08^{\text {th }}$ December, 2021 \\ Published date: $10^{\text {th }}$ December, 2021}

**Corresponding Author: Michael J. Sadowsky, PhD, Professor, Biotechnology Institute, University of Minnesota, 1479 Gortner Ave, 140 Gortner Labs, St. Paul, MN 55108. United States. E-mail: sadowsky@umn.edu

${ }^{* *}$ Corresponding Author: Alexander Khoruts, MD, Department of Medicine, Division of Gastroenterology, Hepatology and Nutrition, 420 Delaware St SE, MMC 36, Minneapolis, MN 55455. United States. E-mail: khoru001@umn.edu

Citation: Sadowsky, M.J., Erickson, R., Wang, P., Hamilton, M.J., Hoo, M., Kaiser, T., Fryxell, K., \& Khoruts, A., (2021). Inactivation of Clostridioides Difficile Spores in Carpeting and Upholstery to Reduce Disease Recurrence in Households and Nursing Care Facilities. J Pub Health Issue Pract 5(2): 189. doi: https://doi.org/10.33790/jphip1100189

Copyright: (C2021, This is an open-access article distributed under the terms of the Creative Commons Attribution License 4.0, which permits unrestricted use, distribution, and reproduction in any medium, provided the original author and source are credited.

\begin{abstract}
Objective: Clostridioides difficile (formerly Clostridium difficile) is among the most reported hospital and community-acquired gastrointestinal infections. Spores produced by this bacterium cause the initial disease and recurrent $C$. difficile infections (rCDI) and survive for long periods in the environment. Killing of $C$. difficile spores in carpeting and upholstery is very challenging without destruction or alteration of the fiber matrix material. In this study, we evaluated a fiber-safe standardized method for inhibiting germination of $C$. difficile spores recovered from in carpeting and upholstery material and thereby reduce the risk of disease incidence in households and nursing care facilities.
\end{abstract}

Methods and Results: In this study, we found that the most effective procedure for the recovery of $C$. difficile spores from shortfiber textile surfaces used an ammonium phosphate-gelatin buffer, stainless steel beads to extract the spores from the carpet samples, and the addition of a heat activation step prior to plating. Virasept $t^{\mathbb{R}}$, a known sporicide containing hydrogen peroxide and peroxyacetic acid, was evaluated for marked reduction of viable $C$. difficile spore number in carpet and fabric models. After a 30 min contact time, application of the sporicide at $\sim 75 \mathrm{ml} \mathrm{m}^{2}$ of carpet (or $51.2 \mathrm{ml} \mathrm{m}^{2}$ upholstery fabric) resulted in a 3-log reduction in germination of five different $C$. difficile spore types (a $\sim 99.9 \%$ decrease), representing five different Nap biotypes. Despite effective inhibition of $C$. difficile spore germination, Virasept treatment did not visibly damage or discolor carpet or fabric fibers.

Conclusions: Results of this study show the potential effectiveness of a robust regimen for the practical treatment of carpeting and upholstery fabric in nursing care facilities, and residential homes to prevent community- and environmentally-acquired reinfection and recurrence of CDI in susceptible individuals.

Keywords: Clostridiodes difficile Recurrence; rCDI; Carpeting; Upholstery Fabric; Spores; Sporicide; Virasept

\section{Introduction}

Clostridioides difficile (formerly Clostridium difficile) is among the most common cause of antibiotic-associated hospital and community-acquired gastrointestinal infections [1-3].

Infection is transmitted to susceptible individuals from the environment by $C$. difficile spores, which are highly resistant to environmental stress and common disinfectants [4-6]. The annual incidence of $C$. difficile infection (CDI) has increased over the past two decades to $\sim 500,000$ cases in the US, resulting in $\sim 29,000$ deaths [3]. Approximately $25 \%$ of these cases were estimated to be due to community acquired infection [3]. Recurrent $C$. difficile infection (rCDI) is especially challenging, and in recent years the incidence of rCDI has risen approximately 4 -fold faster than CDI alone [7]. The probability of further CDI recurrence increases with every episode, and a significant proportion of rCDI patients develop an indefinite syndrome of recurrence [8]. Recurrence is likely driven by residual C. difficile spores, and it is not known whether the main source of these spores is in the patient's own intestinal tract or their immediate environment.

C. difficile spores are actively shed from asymptomatic carriers and individuals with active $C$. difficile infections $[9,10]$. The importance of environmental contamination in transmission of CDI is well recognized in acute and long-term care facilities [11, 12]. Current CDI control guidelines include enhanced cleaning of all potentially contaminated surfaces with $10 \%$ sodium hypochlorite, thorough handwashing, barrier garments for health personnel, and patient isolation [11, 13-15]. Fidaxomicin only modestly lowers the rate of recurrence of CDI over vancomycin [16], and this small benefit may be explained by lower rates of environmental contamination associated with fidaxomicin treatment [17]. CDIdriven diarrhea is characterized by fecal urgency, and most patients suffering from rCDI have episodes of fecal incontinence contributing to environmental contamination [18]. Indeed, C. difficile spores were recovered in most households of rCDI patients examined 
[19-21]. This may explain the high risk of recurrence among fecal microbiota transplantation (FMT) recipients following new antibiotic provocations [22].

Household $C$. difficile contamination is not addressed in the current professional guidelines on rCDI management [23, 24]. While recommendations regarding home environmental control can be found from C. Diff Foundation (https://cdifffoundation.org/ cdiff-infection-homecare/), these focus exclusively on items that can be treated with bleach, including hard surfaces in bathrooms and kitchens and white items launderable with bleach. However, there remains a need to address the many ubiquitous and porus household surfaces that also harbor $C$. difficile spores, including carpeting and fabrics that cannot be treated with bleach without damage. In fact, the contents of home vacuum cleaners were the greatest yield sites for recovery of $C$. difficile spores in FMT recipient households [19].

We hypothesize that spores dwelling in porous household surfaces, including carpeting and upholstery, are important contributors to recurrence of CDI in susceptible individuals. Ultimately, a test of this hypothesis requires an efficient means of decontaminating the households of vulnerable patients. However, disinfection of $C$. difficile from porous surfaces is difficult without the use of strongoxidizing agents, such as sodium hypochlorite-based solutions that may discolor or destroy fiber materials [25]. Therefore, there is a need to develop and validate new sporicides that are not destructive to fabric materials encountered in household environments. Testing such agents also requires a robust method for measuring the abundance of C. difficile spores in carpeting and upholstered surfaces.

Here we describe the use of a sporicide containing hydrogen peroxide and peroxyacetic acid, Virasept, to markedly lower $C$. difficile spore contamination in carpet and fabric models. The results allow us to propose a practical and inexpensive treatment of carpeting that can be used in nursing care facilities and households to prevent recurrence of CDI in susceptible patients.

\section{Materials and Methods}

Clostridium difficile strains: $C$. difficile strains from FMT recipient households were isolated as previously described [26, 27]. Nontoxigenic and reference strains were obtained from the American Type Culture Collection $\left(\mathrm{ATCC}^{\circledR}\right)$. The non-toxigenic ATCC CD 43601 (tcdA ${ }^{-}$, tcdB, $\mathrm{CDTa}^{-} \mathrm{b}^{-}$) was used to initially test experimental parameters. Additional strains examined included: ATCC BAA-1382 (tcdA ${ }^{+}$, tcdB $^{+}$, tcdC $\left.^{+}, \mathrm{CDTa} / \mathrm{b}^{-}\right), \mathrm{ATCC} 43598\left(\mathrm{tcdA}^{-}, \mathrm{tcdB}^{+}, \mathrm{CDTa} / \mathrm{b}^{-}\right)$, the epidemic Nap1 strain RPRE2 $\left(\mathrm{tcdA}^{+}, \mathrm{tcdB}^{+}, \mathrm{tcdC}^{+}, \mathrm{CDTa} / \mathrm{b}^{+}\right)$, and the Nap7 strain RPRE 5-3 (tcdA $\left.{ }^{+}, \operatorname{tcdB}^{+}, \operatorname{tcdC}^{+}, \mathrm{CDTa} / \mathrm{b}^{+}\right)$.

Spore purification: Samples containing $C$. difficile were heat activated for $20 \mathrm{~min}$ at $65^{\circ} \mathrm{C}$ and cultured on taurocholate-cycloserinecefoxitin-fructose agar (TCCFA) [28] anaerobically at $37^{\circ} \mathrm{C}$ for $24-$ $48 \mathrm{~h}$. Individual colonies were subcultured anaerobically on brain heart infusion (BHI) agar (Becton Dickinson, Franklin Lakes, NJ) with $5 \mathrm{~g} / \mathrm{L}$ yeast extract and $0.1 \%(\mathrm{w} / \mathrm{v})$ L-cysteine (BHIS medium) $[26,29-31]$ and incubated for 4-7 d. Anaerobiosis was maintained by using GasPaks (Becton Dickinson). Cells were harvested from agar plates, suspended in $1 \mathrm{~mL}$ sterile deionized water $\left(\mathrm{dH}_{2} \mathrm{O}\right)$, and incubated at $4{ }^{\circ} \mathrm{C}$ for $12-24 \mathrm{~h}$ to induce sporulation. The suspension was added to a $50 \%(\mathrm{w} / \mathrm{v})$ sucrose solution and spores were pelleted by centrifugation in a swinging bucket S-4-104 rotor (Eppendorf 5810R Centrifuge) at $3200 \mathrm{rpm}$ for $20 \mathrm{~min}$ at $4^{\circ} \mathrm{C}$ [27]. Spore pellets were washed 5-times in ice-cold $\mathrm{dH}_{2} \mathrm{O}$, resuspended in $\mathrm{dH}_{2} \mathrm{O}$, and stored at $4^{\circ} \mathrm{C}$ until used.

Spore purity was determined by using a Petroff-Hausser counting chamber and a Zeiss Standard 10 microscope (Carl Zeiss AG, Oberkochen, Germany). Spore stocks were determined to contain $\geq$ 95\% spores and were suspended in phosphate buffered saline (PBS), containing $137 \mathrm{mM} \mathrm{NaCl}, 10 \mathrm{mM} \mathrm{Na}_{2} \mathrm{HPO} 4,1.8 \mathrm{mM} \mathrm{KH_{2 } P O} 4,2.7$ $\mathrm{mM} \mathrm{KCl}$, and $0.01 \%$ tween $80, \mathrm{pH} 7.4$ ) prior to application. Spore's stocks were kept at $4^{\circ} \mathrm{C}$ and were replaced at $<4$-month intervals.

Sporicidal solution: Virasept ${ }^{\circledR}$ was obtained from Amazon.com. The active ingredients are hydrogen peroxide (3.13\%), octanoic acid $(0.099 \%)$, and peroxyacetic acid $(0.05 \%)$. The sporicide is known to be an effective disinfectant on hard, non-porous, surfaces such as plastics, porcelain, stainless steel, and wood according to the Association of Official Agricultural Chemists (AOAC) Sporicidal Activity of Disinfectants Method (996.04).

Carpet and upholstery fabric: Carpet tested was Citation Firecracker Level Loop Carpet (fiber material: 100\% BCF Olefin, pile height: 4/32, attached pad). The upholstery fabric tested was $100 \%$ polyester, light upholstery grade. Prior to use, fabric was washed in a solution of $0.1 \%$ tween 80 , rinsed in $\mathrm{dH}_{2} \mathrm{O}$, and air dried overnight.

Evaluation of assay parameters: Test parameters for the extraction procedure were partially adapted from the American Society for Testing and Materials (ASTM)'s State Test Method for Quantitative Assessment of Sanitizing Solutions for Carpet (CASTM Int'1 E296614). Approximately $2-5 \times 10^{7}$ purified spores were suspended in

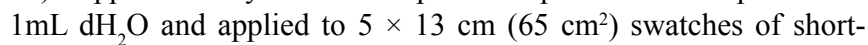
fiber, industrial, carpeting using a glass chromatography sprayer (Sigma Aldrich, St. Louis, MO). Spores were applied at 1-2 psi and $8 \mathrm{~cm}$ from the surface, and swatches were air dried for $20 \mathrm{~min}$. After drying, varying volumes $\left(0.8\right.$ to $\left.30.8 \mathrm{ml} \mathrm{m}^{-2}\right)$ of the sporicide were applied evenly with a glass chromatography sprayer onto an inoculated carpet square. Controls received only $\mathrm{dH}_{2} \mathrm{O}$.

After the desired contact time, and in order to extract spores, carpet swatches were placed into sterile, wide mouthed, $160 \mathrm{~mL}$ milk dilution bottles containing $125 \mathrm{ml}$ of sterilized buffer and 40 g $6 \mathrm{~mm}$ stainless steel beads (Grade 25 chrome steel ball bearings). Four spore extraction buffers were tested for efficacy: ammonium phosphate $\left(0.1 \mathrm{M}\right.$ ammonium phosphate $\left[\left(\mathrm{NH}_{4}\right)_{2} \mathrm{HPO}_{4}\right], \mathrm{pH} 7.4$, containing $0.1 \%$ partially hydrolyzed gelatin, and $0.01 \%$ Tween 80 (APB) [32]) ; $0.1 \%$ trisodium pyrophosphate (TSPP), $\mathrm{pH} 7.0$; $0.58 \% \mathrm{NaCl}$ containing $0.01 \%$ Tween 80 ; and PBS containing $0.01 \%$ tween 80. Carpet samples were placed into milk bottles lengthwise and arranged so that the beads could freely move along the carpet fiber surfaces during the extraction process. Bottles were subjected to vigorous agitation (1.5" stroke, $280 \mathrm{osc} / \mathrm{min}$ ) for $60 \mathrm{~min}$ on a reciprocating shaker (Eberbach Corp, Ann Arbor, MI.).

Extraction buffer aliquots were incubated for $20 \mathrm{~min}$ at $65^{\circ} \mathrm{C}$ to heat activate the spores, and serial dilutions of the sample were plated onto BHIS containing $0.1 \%(\mathrm{w} / \mathrm{v})$ taurocholic acid (TCA) media to determine spore germination potential. Plates were incubated at $37^{\circ} \mathrm{C}$ for 18-48 h under anaerobic conditions and colonies were counted.

Triplicate sporicidal test volumes were run, and results were compared to controls sprayed with $\mathrm{dH}_{2} \mathrm{O}$ to determine the sporicidal solution's relative ability to inhibit germination. Linear portions of the dose-response curve were averaged, curves were log-transformed, and $\log$ reduction values calculated. The trend lines of the semi-log plots were extrapolated to determine the sporicidal volumes needed for 1,2 , or $3-\log$ reductions $(90 \%, 99 \%$, and $99.9 \%$ inhibition) in germination of spores. This methodology was repeated to spores from five $C$. difficile test strains to determine individual sensitivity to Virasept on short-fiber carpet and upholstery fabric.

Determination of contact times of sporicidal solution: The contact time assay used the same methodology as did the volume assay. The volume of sporicidal solution used was calculated to produce a 3-log reduction in viable spores for each strain and initial studies tested contact times of $1,2.5,5,10,20$, and $30 \mathrm{~min}$. The resulting data was $\log$ transformed to determine contact times required for $\log$ reduction in spore viability.

Statistical analysis: Log reduction times for germination inhibition by different volumes of applied sporicidal solution were determined from semi-log plots of viable spore counts/volume solution applied 
$\left(\mathrm{ml} \mathrm{m}^{-2}\right)$. The slope of the resulting curve was used to calculate extrapolated volumes for 1-, 2-, and 3-log reductions in viable spore counts/30 min contact time. Statistical significance was tested by ANOVA using pairwise comparisons at $\alpha=0.05$, by Tukey's honestly significant difference (HSD) post hoc test, and unpaired, two tailed t-tests with $95 \%$ confidence intervals.

\section{Results}

The goal of this study was to develop and utilize a method to reduce risk of recurrence of CDI due to $C$. difficile spore contamination from textile surfaces in households and nursing care facilities. The immediate objectives of this study were to: 1) develop methods to quantify spores in fibrous materials and 2) evaluate the sporicidal activity of Virasept on short-fiber carpet and upholstery fabric, without destruction or discoloration of the matrices.
Optimized extraction procedures from short-fiber carpet samples: The efficacy of various buffers used to extract spores from carpet samples are shown in Figure 1A. Extraction of spores from carpet with APB buffer resulted in significantly greater recovery of spores compared to $0.9 \% \mathrm{NaCl}$, TSPP, or PBS buffers $(\mathrm{p}<0.0001)$. Furthermore, the use of APB with $0.1 \%$ hydrolyzed gelatin was less variable compared to the other buffers tested (Figure 1A).

As seen in Figure 1B, the addition of a $65^{\circ} \mathrm{C}$ heat activation step significantly increased the apparent extraction efficiency by $39.8 \% \%$ $(\mathrm{p}=0.0013)$. There was an incremental increase in extraction efficiency by extending the shaking time from $30 \mathrm{~min}$ to $1 \mathrm{~h}$, and increasing the initial quantity of applied spores, although these results were not significantly different (Figure 1C and 1D).
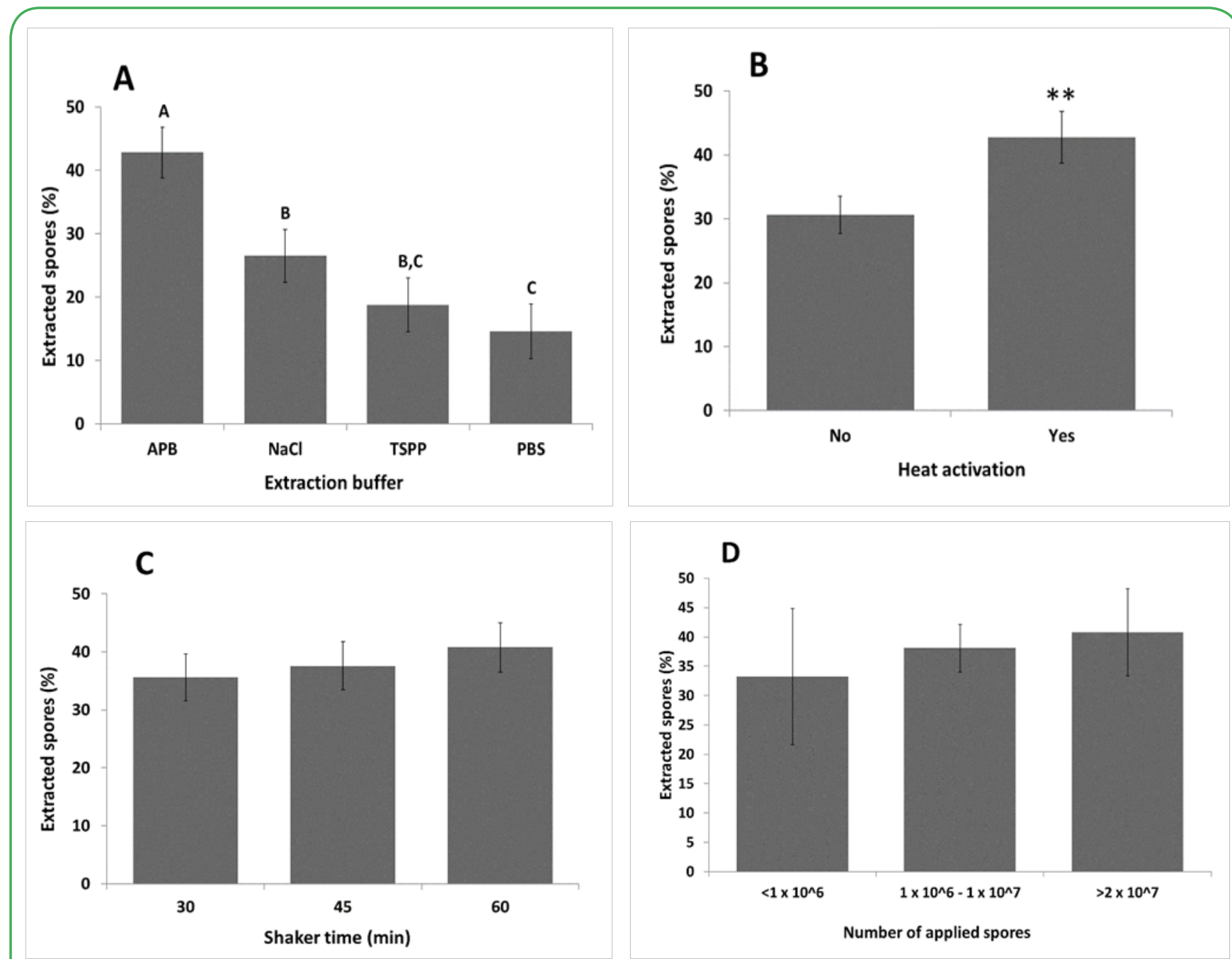

Figure 1, Sadowsky et al.

Figure 1: (A) Direct comparison of different buffers (NaCl, PBS. TSPP, and APB) used in the extraction procedure from shortfiber carpet. Error bars indicate standard error of the mean (SEM). (B) Percent $C$. difficile spores extracted with and without a heat activation of $65^{\circ} \mathrm{C}$ for $20 \mathrm{~min}$ prior to plating. (C) Percentage of C. difficile spores extracted based on horizontal shaker extraction time (min). (D) Percentages of $C$. difficile spores extracted based on quantity of initial spore application to carpet sample.

Virasept effectively inhibits germination of $C$. difficile spores recovered from carpeting: The efficacy of the sporicidal solution was tested by the carpet assay outlined above. Results in Figure $2 \mathrm{~A}$ and $2 \mathrm{~B}$ show that increased volumes of applied sporicide led to corresponding decreases in viable $C$. difficile spores following the extraction protocol. This effect followed closely to a dose-response sigmoidal curve for all strains (Figure 1D) and the slopes of the linear portion of the dose-response curves (Figure 1C) allowed for calculation of volumes of Virasept needed to give 1, 2, and 3-Log reductions in viable spores from multiple $C$. difficile strains (Table 1). Moreover, the tested sporicide effectively inactivated spores from several different $C$. difficile strains, including the Nap1 epidemic strain (Table 1), even at relatively low volumes $\left(\sim 70 \mathrm{ml} \mathrm{m}^{2}\right.$ for carpet or $\sim 45 \mathrm{ml} \mathrm{m}^{2}$ for upholstered fabric). 


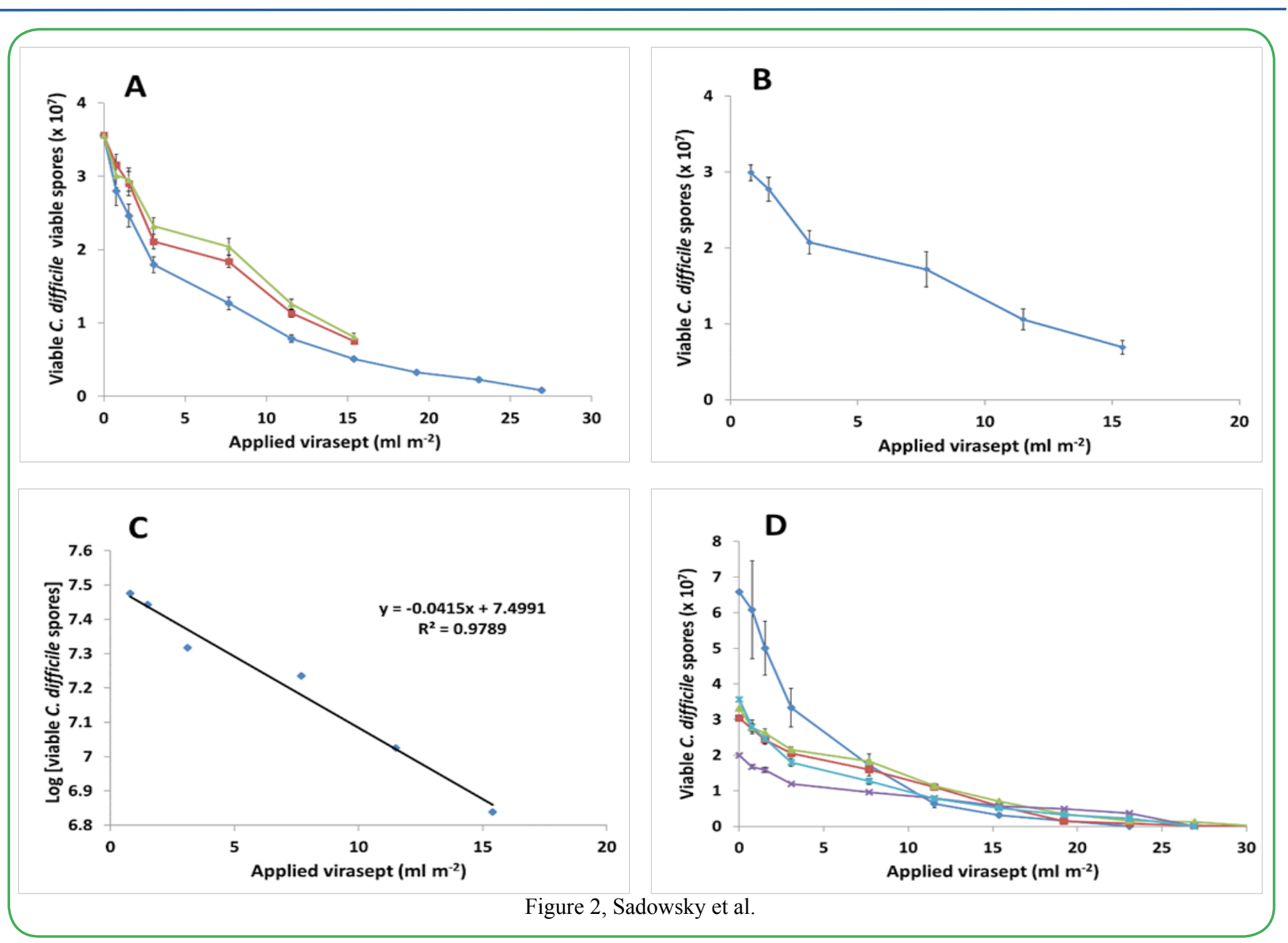

Figure 2. Influence of Virasept volume on germination of purified C. difficile spores. (A) Germination of ATCC 43598 spores after exposure to varying volumes of Virasept following a $30 \mathrm{~min}$ contact time. Three biological replicates were used per treatment group. Error bars indicate SEM. (B) Average of the linear portions of three biological replicates for ATCC strain 43598 using varying volumes of Virasept. (C) Semi-log plot of spore inhibition based on $\log 10$ spore counts per volume of Virasept following a $30 \mathrm{~min}$ contact time. The slope of the curve was used to calculate the log reduction values for Virasept at a 30 min contact time. (D) Efficacy of Virasept on inhibiting germination of five test strains of $C$. difficile ( $\diamond$ - ATCC 43601, $\square$ - Nap1 (RPRE2), $\Delta$ - ATCC 1382), *- Nap7 (RPRE 5-3), X- ATCC 43598)

\begin{tabular}{|c|c|c|c|c|c|c|c|}
\hline \multicolumn{3}{|c|}{ Reduction Efficacy } & \multicolumn{5}{|c|}{ C. difficile strain tested } \\
\hline $\log 10$ & $\%$ & Matrix & A $\underset{43601}{\mathrm{~T}} \mathrm{C}$ C & $\begin{array}{c}\text { Nap1 } \\
\text { (RPRE2) }\end{array}$ & $\begin{array}{l}\text { A } \mathrm{T} \text { C } \mathrm{C} \\
\text { BAA- } 1382\end{array}$ & 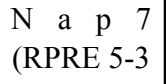 & $\begin{array}{l}\text { A } \mathrm{T} \mathrm{C} \mathrm{C} \\
43598\end{array}$ \\
\hline \multirow[t]{2}{*}{1} & \multirow[t]{2}{*}{90.0} & Carpet & $10.1^{*}$ & 21.6 & 24.4 & 22.1 & 22.8 \\
\hline & & Upholstery & 13.2 & 15.1 & 13.4 & 15.5 & 18.2 \\
\hline \multirow[t]{2}{*}{2} & \multirow[t]{2}{*}{99.0} & Carpet & 20.6 & 43.7 & 49.1 & 45.5 & 34.7 \\
\hline & & Upholstery & 25.8 & 28.4 & 26.5 & 29.9 & 71.0 \\
\hline \multirow[t]{2}{*}{3} & \multirow[t]{2}{*}{99.9} & Carpet & 31.2 & 65.8 & 73.7 & 69.0 & 71.0 \\
\hline & & Upholstery & 38.4 & 41.7 & 39.6 & 44.3 & 51.2 \\
\hline \multicolumn{8}{|c|}{$\begin{array}{l}\text { *Values are } \mathrm{mL} / \mathrm{m} 2 \text { of sporicide used to obtain indicated percent reductions. Average values } \pm \mathrm{SEM} \text { for } \\
\text { each indicated test strain. }\end{array}$} \\
\hline
\end{tabular}

Contact Time affects efficacy of sporicidal solutions: The effect of contact time on the efficacy of the sporicide was tested using volumes inhibiting $99.9 \%$ of spore germination. Results in Figure $3 \mathrm{~A}$ show that contact time of the sporicidal solution required to kill spores of $C$. difficile strains also followed a dose-response-type curve, with significant differences between the contact time points ( $\mathrm{p}$ $=0.0346)$ (Figure 3B).

$\mathrm{J}$ Pub Health Issue Pract

Volume 5. 2021. 189
The linear portion of the curves were used to calculate spore killing efficacy throughout the exposure assay (Figure 3C), and indicated that this volume of sporicidal solution (Table 1) was only $97.2 \%$ effective after $1 \mathrm{~min}$. However, it was $99.0 \%$ and 99.3\% effective after 20 - and 30-min contact times, respectively. 


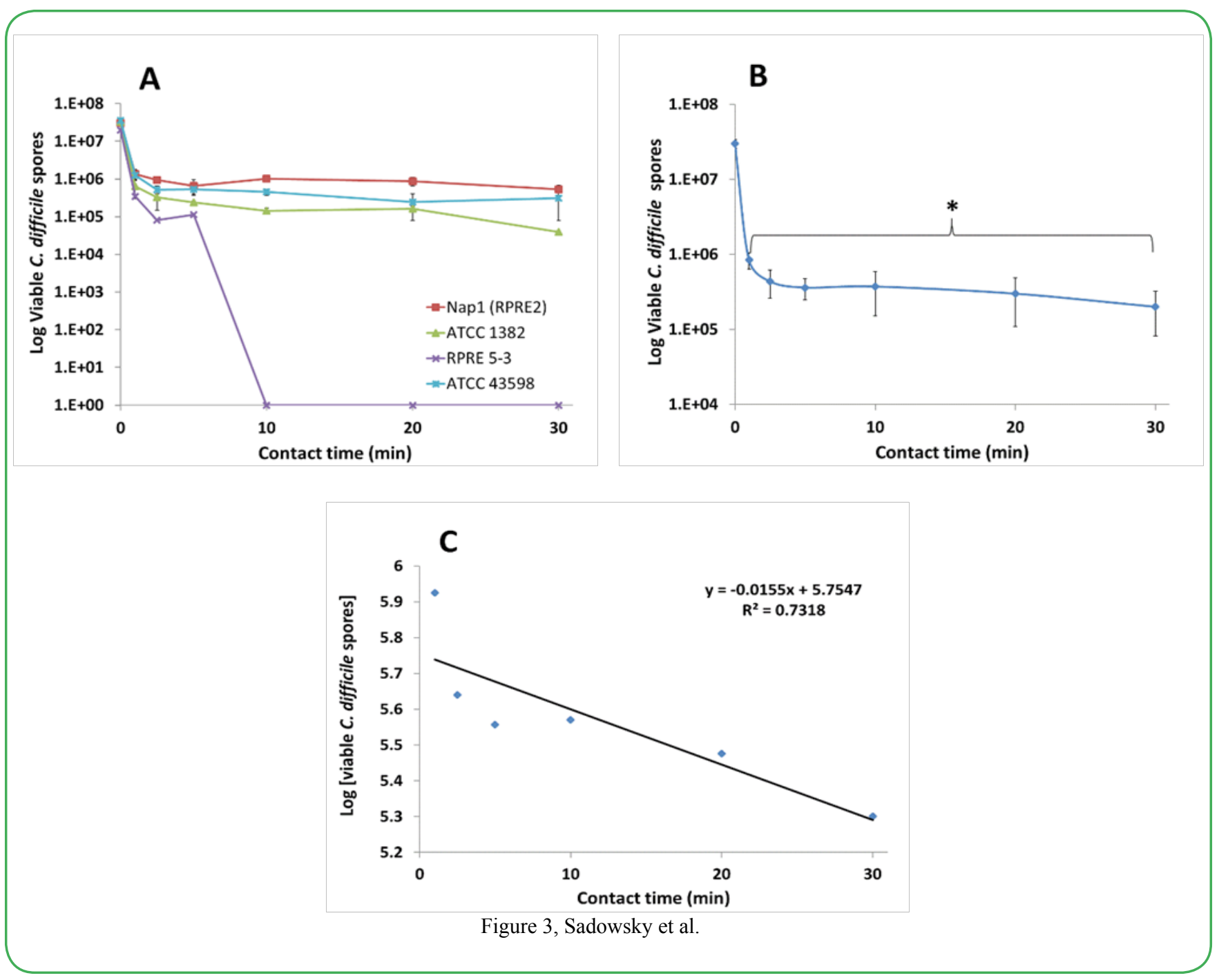

Figure 3. Influence of contact time on efficacy of Virasept on inhibiting germination of $C$. difficile spores. (A) Viability of $C$. difficile test spore strains after exposure to a fixed volume of Virasept calculated to inhibit $99.9 \%$ of $C$. difficile after 30 minute contact time at various time points. Data represents $\mathrm{N}=3$. Error bars indicate SEM. Legend: $\square$ - Nap1 (RPRE2), $\Delta$ - ATCC 1382, *- Nap7 (RPRE 5-3), X- ATCC 43598). (B) Average of the linear portions of the triplicate assays of $C$. difficile strains at varying time points. Error bars indicate the SEM between replicates of each strain. Statistical significance base on an unpaired, two tail t-test with $95 \%$ confidence interval $(\mathrm{p}=0.0346)$. (C) Semi-log plot of calculated viable spores following the application of Virasept at a fixed volume for various time points. Slope of the curve was used to calculate the log reduction values for Virasept contact time at various time points for a volume of Virasept calculated to inhibit $99.9 \%$ of spore germination in $30 \mathrm{~min}$.

\section{Discussion}

Recently, a large proportion of $C$. difficile infections are due to community acquisition [21,33-35]. Consequently, there is a need for an easily applied solution to eradicate bacterial spores in home and nursing care settings. This need is often expressed by patients themselves who realize that their home setting may be contaminated with $C$. difficile spores. Based on other disease models, it seems reasonable that reduction in the environmental load of $C$. difficile spores will result in reduced rates of disease recurrence. While there is no direct evidence for this correlation with rCDI, it is often recommended that the patient's immediate environment be decontaminated with commonly-used hospital cleaning compounds, such as quaternary ammonium salt-based solutions [36]. Alarmingly, however, there are now reports that some of these compounds may stimulate spore germination [13]. Consequently, it is now recommended that sporicidal solutions containing hydrogen peroxide or sodium hypochlorite be used to decontaminate solid surfaces [11, 37-40].
Based on our initial analyses, however, the vacuum cleaner and not solid surfaces was found to be the primary household environment contaminated with $C$. difficile spores [19]. This suggested that spores released by symptomatic and asymptomatic carriers made their way onto environmental surfaces, including carpeting [41]. Thus, carpeting may be a reservoir for environmental $C$. difficile. Moreover, since carpeting is often not sufficiently cleaned, and most effective sporicides cannot be used on fibrous materials without decolorization or destruction, this may be one cause of rCDI in household and nursing care settings.

Consequently, the aim of this current study was to determine and optimize testing parameters to evaluate the efficacy of chemical agents on the reduction in the viability of $C$. difficile spores found in carpet, without matrix material destruction or discoloration. This methodology was subsequently used to test the ability of a commercial sporicide, Virasept, to inactivate spores of pathogenic $C$. difficile strains. Bacterial spores carry a charge and have surface 
hydrophobicity, due in part to collagen-like BclA1 orthologs in the C. difficile exosporium [42-45], and likely bind to charged carpet fibers. We hypothesized that disruption of this charge and reduction of surface tension by using a buffered surfactant would facilitate the release of spores from the carpet matrix. The APB buffer with $0.1 \%$ hydrolyzed gelatin supplemented with Tween 80 resulted in the highest levels of extracted and viable $C$. difficile spores of the tested buffer solutions. Also, the addition of a $65^{\circ} \mathrm{C}$ heat activation step, which has been shown to be necessary to ensure high levels of Clostridium spore germination in culture [46], and extension of the extraction time resulted in the greatest possible extraction efficiencies for this assay.

In addition to the above optimization parameters, we also evaluated bead size and composition, sprayer pressure and distance to spray carpet fibers, and drying time prior to assay. Based on these initial studies, glass beads (2- and 3-mm solid glass) were found to be inefficient at extractions relative to stainless steel beads, mostly due to bead size and weight (data not shown). It was also determined that sprayer pressures less than 1 PSI were too low to create adequate misting for an even application of spores, whereas pressures $>2$ discharged the spore solution too quickly to evenly coat the carpet samples. Moreover, application with the sprayer closer than $\sim 7 \mathrm{~cm}$ resulted in uneven spore distribution and oversaturation to the applied areas, and $>9 \mathrm{~cm}$ resulted in volume loss past the edges of the carpet. Thus, an application distance of $8 \mathrm{~cm}$ was chosen for all studies. Lastly, it was determined that 20 min was sufficient to completely air dry the $2 \mathrm{ml}$ spore application under ambient conditions.

Previous studies have shown that the two most important factors affecting the efficacy of antimicrobials are the quantity of active ingredient and contact time [25, 47]. We showed that even relatively low volumes of the tested sporicide resulted in 3-log reductions in spore counts using several different $C$. difficile strains, including the Nap1 epidemic strain (Table 1). This result is consistent with reports that active ingredients in Virasept, hydrogen peroxide and peroxyacetic acid, are effective inhibitors of $C$. difficile spore germination on hard surfaces (should be 48 Barbut et al., 2009). Based on our results, an application volume of $75 \mathrm{ml} / \mathrm{m} 2$ of Virasept, diluted in $\mathrm{dH}_{2} 0$, spread evenly on a carpeted surface (or $55 \mathrm{ml} \mathrm{m}^{2}$ for upholstery fabric) would be sufficient to inactivate $>99.9 \%$ of viable $C$. difficile spores. It should be noted, however, that while we did not specifically test whether Virasept killed spores, this product is a known sporicide, and the reduction in spore germination after treatment and extraction from carpet and upholstery is likely due to inactivation of spores.

Since contact time has been reported to be important when inhibiting spore germination [47], and since real-world carpet cleaning processes can vary between users, we examined sporicidal activity in carpet vs contact time. Even at relatively high application volumes, contact time had significant impact on the efficacy of the sporicidal solution. Nevertheless, our results are in general agreement with previous reports that indicated that contact time plays a significant role in the ability of sporicidal solutions to inhibit germination of C. difficile spores [25]. The volume needed for a 3-log reduction in viable $C$. difficile spores at a 30 min contact time is also well below the typical volumes of antimicrobials that would be applied during the normal course of the carpet disinfection process [49]. Moreover, at this volume and contact time, the Virasept solution is below any observable threshold for discoloration or visible damage of the carpet fibers. It is important to note that one limitation of this current study was our lack of use of a quantifiable method to determine Viraseptinduced color changes or fiber damage to carpeting and upholstery fabric. Consequently, future studies need to address this limitation and potential problem by using ISO/AATCC grey scales, as described in AATCC EP1[50], or instrumental color analysis as discussed in AATCC EP6-2016e [51] and by microscopy.
Previous studies have indicated that although purified spores alone can act as a model for testing the efficacy of sporicidal compounds on $C$. difficile strains [25, 53], organic loading in carpeting will also need to be examined in subsequent testing $[52,53]$. Additionally, the difference in sensitivity between spores applied to the carpet surface, and those located deeper in carpet fibers or in the backing-material (foam, urethane, or jute) will need to be addressed for both the extraction and testing methodologies. Elimination of these variables will provide a clearer understanding of how this disinfectant performs in real operational conditions to reduce reinfection rates and recurrence of CDI.

Taken together, however, our data nevertheless allow us to propose a robust regimen for the practical treatment of carpeting and upholstery fabric in nursing care facilities and residential homes to reduce the incidence of environment-acquired reinfection and recurrence of CDI in susceptible individuals. Reducing and potentially eliminating his under-appreciated environmental reservoir may lead to a reduction in rCDI rates and needs to be further examined in a controlled study.

Competing interests: The authors report no conflict of interest. List of abbreviations:

CDI: C. difficile infection

rCDI: recurrent $C$. difficile infection

FMT: fecal microbiota transplantation

ATCC: American Type Culture Collection

AOAC: Association of Official Agricultural Chemists

ANOVA: Analysis of Variance

APB: ammonium phosphate buffer

ASTM: American Society for Testing and Materials

BHI: brain heart infusion

PBS: phosphate buffered saline

TCA: taurocholic acid

TCCFA: taurocholate-cycloserine-cefoxitin-fructose agar

TSPP: trisodium pyrophosphate

Tukey's HSD: Tukey's honestly significant difference

Acknowledgement: This study was supported, in part, by grant NIH 1R21-AI114722-01 and by the philanthropic support of Achieving Cures Together and the Hubbard Foundation.

\section{References:}

1. Kaatz, G. W., Gitlin, S. D., Schaberg, D. R., Wilson, K. H., Kauffman, C. A., Seo, S. M., \& Fekety, R. (1988). Acquisition of Clostridium difficile from the Hospital Environment. American Journal of Epidemiology, 127(6), 1289-1294.

2. Kelly, C. P., \& LaMont, J. T. (2008). Clostridium difficile - More difficult than ever. New England Journal of Medicine, 359(18).

3. Lessa, F. C., Mu, Y., Bamberg, W. M., Beldavs, Z. G., Dumyati, G. K., Dunn, J. R., . . McDonald, L. C. (2015). Burden of Clostridium difficile Infection in the United States. New England Journal of Medicine, 372(9), 825-834.

4. Kramer, A., Schwebke, I., \& Kampf, G. (2006). How long do nosocomial pathogens persist on inanimate surfaces? A systematic review. BMC Infectious Diseases, 6, 130. doi:10.1186/1471-2334-6-130

5. Brite, J., McMillen, T., Robilotti, E., Sun, J., Chow, H. Y., Stell, F., . . Kamboj, M. (2018). Effectiveness of ultraviolet disinfection in reducing hospital-acquired Clostridium difficile and vancomycin-resistant Enterococcus on a bone marrow transplant unit. Infection Control and Hospital Epidemiology, 39(11), 1301-1306. 
6. Buckley, D., Dharmasena, M., Wang, H., Huang, J., Adams, J., Pettigrew, C., . . Jiang, X. (2021). Efficacy of novel aqueous photo-chlorine dioxide against a human norovirus surrogate, bacteriophage MS2 and Clostridium difficile endospores, in suspension, on stainless steel and under greenhouse conditions. Journal of Applied Microbiology, 130(5), 1531-1545.

7. Ma, G. K., Brensinger, C. M., Wu, Q., \& Lewis, J. D. (2017). Increasing Incidence of Multiply Recurrent Clostridium difficile Infection in the United States: A Cohort Study. Annals of Internal Medicine, 167(3), 152-158. doi:10.7326/M16-2733.

8. Borody, T. J., \& Khoruts, A. (2012). Fecal microbiota transplantation and emerging applications. Nat Rev Gastroenterol Hepatol, 9(2), 88-96. doi:10.1038/nrgastro.2011.244.

9. Riggs, M. M., Sethi, A. K., Zabarsky, T. F., Eckstein, E. C., Jump, R. L., \& Donskey, C. J. (2007). Asymptomatic carriers are a potential source for transmission of epidemic and nonepidemic Clostridium difficile strains among long-term care facility residents. Clinical Infectious Diseases, 45(8), 992-998. doi:10.1086/521854.

10. Sethi, A. K., Al-Nassir, W. N., Nerandzic, M. M., Bobulsky, G. S., \& Donskey, C. J. (2010). Persistence of skin contamination and environmental shedding of Clostridium difficile during and after treatment of $C$. difficile infection. Infection Control and Hospital Epidemiology, 31(1), 21-27. doi:10.1086/649016.

11. Dubberke, E. R., Carling, P., Carrico, R., Donskey, C. J., Loo, V. G., McDonald, L. C., . . Gerding, D. N. (2014). Strategies to prevent Clostridium difficile infections in acute care hospitals: 2014 Update. Infect Control Hosp Epidemiol, 35(6), 628-645. doi:10.1086/676023.

12. Srinivasa, V. R., Hariri, R., Frank, L. R., Kingsley, L., Magee, E., Pokrywka, M., \& Yassin, M. H. (2019). Hospital-associated Clostridium difficile infection and reservoirs within the hospital environment. American Journal of Infection Control, 47(7), 780-785.

13. Gerding, D. N., Muto, C.A., \& Owens, R. C., Jr. (2008). Measures to control and prevent Clostridium difficile infection. Clinical Infectious Diseases, 46 Suppl 1, S43-49. doi:10.1086/521861.

14. Vonberg, R. P., Kuijper, E. J., Wilcox, M. H., Barbut, F., Tull, P., Gastmeier, P., ... Wiuff, C. (2008). Infection control measures to limit the spread of Clostridium difficile. Clin Microbiol Infect, 14 Suppl 5: p. 2-20.

15. Barbut, F. (2015). How to eradicate Clostridium difficile from the environment. Journal of Hospital Infection, 89(4), 287-295.

16. Louie, T. J., Miller, M. A., Mullane, K. M., Weiss, K., Lentnek, A., Golan, Y., . . . Group, O. P. T. C. S. (2011). Fidaxomicin versus vancomycin for Clostridium difficile infection. New England Journal of Medicine, 364(5), 422-431. doi:10.1056/ NEJMoa0910812.

17. Biswas, J. S., Patel, A., Otter, J. A., Wade, P., Newsholme, W., van Kleef, E., \& Goldenberg, S. D. (2015). Reduction in Clostridium difficile environmental contamination by hospitalized patients treated with fidaxomicin. Journal of Hospital Infection, 90(3), 267-270. doi:10.1016/j.jhin.2015.01.015.

18. Khoruts, A., Rank, K. M., Newman, K. M., Viskocil, K., Vaughn, B. P., Hamilton, M. J., \& Sadowsky, M. J. (2016). Inflammatory Bowel Disease Affects the Outcome of Fecal Microbiota Transplantation for Recurrent Clostridium difficile Infection. Clinical Gastroenterology and Hepatology. doi:10.1016/j. cgh.2016.02.018.

19. Shaughnessy, M. K., Bobr, A., Kuskowski, M. A., Johnston, B. D., Sadowsky, M. J., Khoruts, A., \& Johnson, J. R. (2016). Environmental Contamination in Households of Patients with Recurrent Clostridium difficile Infection. Applied and Environmental Microbiology, 82(9), 2686-2692. doi:10.1128/ AEM.03888-15.

J Pub Health Issue Pract

Volume 5. 2021. 189
20. Loo, V.G., Brassard, P. \& Miller, M.A. (2016). Household Transmission of Clostridium difficile to Family Members and Domestic Pets. Infection Control and Hospital Epidemiology, 37(11): p. 1342-1348.

21. Miller, A. C., Segre, A. M., Pemmaraju, S. V., Sewell, D. K., \& Polgreen, P. M. (2020). Association of Household Exposure to Primary Clostridioides difficile Infection With Secondary Infection in Family Members. JAMA Netw Open, 3(6), e208925. doi:10.1001/jamanetworkopen.2020.8925.

22. Brandt, L. J., Aroniadis, O. C., Mellow, M., Kanatzar, A., Kelly, C., Park, T., . . . Surawicz, C. (2012). Long-term follow-up of colonoscopic fecal microbiota transplant for recurrent Clostridium difficile infection. American Journal of Gastroenterology, 107(7), 1079-1087. doi:10.1038/ajg.2012.60.

23. Cohen, S. H., Gerding, D. N., Johnson, S., Kelly, C. P., Loo, V. G., McDonald, L. C., ... Infectious Diseases Society of, A. (2010). Clinical practice guidelines for Clostridium difficile infection in adults: 2010 update by the society for healthcare epidemiology of America (SHEA) and the infectious diseases society of America (IDSA). Infection Control and Hospital Epidemiology, 31(5), 431-455. doi:10.1086/651706.

24. Surawicz, C. M., Brandt, L. J., Binion, D. G., Ananthakrishnan, A. N., Curry, S. R., Gilligan, P. H., . . Zuckerbraun, B. S. (2013). Guidelines for diagnosis, treatment, and prevention of Clostridium difficile infections. American Journal of Gastroenterology, 108(4), 478-498; quiz 499. doi:10.1038/ ajg.2013.4.

25. Lawley, T. D., Clare, S., Deakin, L. J., Goulding, D., Yen, J. L., Raisen, C., . . . Dougan, G. (2010). Use of purified Clostridium difficile spores to facilitate evaluation of health care disinfection regimens. Applied and Environmental Microbiology, 76(20), 6895-6900. doi:10.1128/AEM.00718-10.

26. Sorg, J. A., \& Dineen, S. S. (2009). Laboratory maintenance of Clostridium difficile. Curr Protoc Microbiol, Chapter 9, Unit9A 1. doi:10.1002/9780471729259.mc09a01s12.

27. Weingarden, A. R., Dosa, P. I., DeWinter, E., Steer, C. J., Shaughnessy, M. K., Johnson, J. R., . . . Sadowsky, M. J. (2016). Changes in Colonic Bile Acid Composition following Fecal Microbiota Transplantation Are Sufficient to Control Clostridium difficile Germination and Growth. Plos One, 11(1), e0147210. doi:10.1371/journal.pone.0147210.

28. Bliss, D. Z., Johnson, S., Clabots, C. R., Savik, K., \& Gerding, D. N. (1997). Comparison of cycloserine-cefoxitinfructose agar (CCFA) and taurocholate-CCFA for recovery of Clostridium difficile during surveillance of hospitalized patients. Diagn Microbiol Infect Dis, 29(1), 1-4. doi:10.1016/ s0732-8893(97)00113-2.

29. Allen, C. A., Babakhani, F., Sears, P., Nguyen, L., \& Sorg, J. A. (2013). Both fidaxomicin and vancomycin inhibit outgrowth of Clostridium difficile spores. Antimicrobial Agents and Chemotherapy, 57(1), 664-667. doi:10.1128/AAC.01611-12.

30. Francis, M. B., Allen, C. A., Shrestha, R., \& Sorg, J. A. (2013). Bile acid recognition by the Clostridium difficile germinant receptor, CspC, is important for establishing infection. PLoS Pathogens, 9(5), e1003356. doi:10.1371/journal.ppat.1003356.

31. Francis, M. B., Allen, C. A., \& Sorg, J. A. (2015). Spore Cortex Hydrolysis Precedes Dipicolinic Acid Release during Clostridium difficile Spore Germination. Journal of Bacteriology, 197(14), 2276-2283. doi:10.1128/JB.02575-14.

32. Kingsley, M. T., \& Bohlool, B. B. (1981). Release of Rhizobium spp. from Tropical Soils and Recovery for Immunofluorescence Enumeration. Applied and Environmental Microbiology, 42(2), 241-248. 
33. Deshpande, A., Pasupuleti, V., Thota, P., Pant, C., Rolston, D. D. K., Sferra, T. J., . . . Donskey, C. J. (2013). CommunityAssociated Clostridium difficile Infection and Antibiotics: A Meta-Analysis. Journal of Investigative Medicine, 61(4), 775775.

34. DiDiodato, G., \& Fruchter, L. (2019). Antibiotic exposure and risk of community-associated Clostridium difficile infection: A self-controlled case series analysis. American Journal of Infection Control, 47(1), 9-12.

35. Taori, S. K., Wroe, A., Hardie, A., Gibb, A. P., \& Poxton, I. R. (2014). A prospective study of community-associated Clostridium difficile infections: The role of antibiotics and coinfections. Journal of Infection, 69(2), 134-144.

36. Kenters, N., Huijskens, E. G. W., de Wit, S. C. J., Sanders, I. G. J. M., van Rosmalen, J., Kuijper, E. J., \& Voss, A. (2017). Effectiveness of various cleaning and disinfectant products on Clostridium difficile spores of PCR ribotypes 010, 014 and 027. Antimicrobial Resistance and Infection Control, 6.

37. Rutala, W. A., \& Weber, D. J. (1997). Uses of inorganic hypochlorite (bleach) in health-care facilities. Clinical Microbiology Reviews, 10(4), 597-610.

38. Boyce, J. M., Havill, N. L., Otter, J. A., McDonald, L. C., Adams, N. M., Cooper, T., . . Noble-Wang, J. (2008). Impact of hydrogen peroxide vapor room decontamination on Clostridium difficile environmental contamination and transmission in a healthcare setting. Infection Control and Hospital Epidemiology, 29(8), 723-729. doi:10.1086/589906

39. Dyer, C., Hutt, L. P., Burky, R., \& Joshi, L. T. (2019). Biocide Resistance and Transmission of Clostridium difficile Spores Spiked onto Clinical Surfaces from an American Health Care Facility. Applied and Environmental Microbiology, 85(17).

40. Goda, H., Yamaoka, H., Nakayama-Imaohji, H., Kawata, H., Horiuchi, I., Fujita, Y., . . Kuwahara, T. (2017). Microbicidal effects of weakly acidified chlorous acid water against feline calicivirus and Clostridium difficile spores under protein-rich conditions. Plos One, 12(5).

41. Donskey, C. J. (2010). Preventing transmission of Clostridium difficile: is the answer blowing in the wind? Clinical Infectious Diseases, 50(11), 1458-1461. doi:10.1086/652649

42. Brahmbhatt, T. N., Janes, B. K., Stibitz, E. S., Darnell, S. C., Sanz, P., Rasmussen, S. B., \& O'Brien, A. D. (2007). Bacillus anthracis exosporium protein BclA affects spore germination, interaction with extracellular matrix proteins, and hydrophobicity. Infection and Immunity, 75(11), 5233-5239. doi:10.1128/IAI.00660-07.

43. Abhyankar, W., Hossain, A. H., Djajasaputra, A., Permpoonpattana, P., Ter Beek, A., Dekker, H. L., . . . de Koster, C. G. (2013). In pursuit of protein targets: proteomic characterization of bacterial spore outer layers. Journal of Proteome Research, 12(10), 4507-4521. doi:10.1021/ pr4005629.
44. Pizarro-Guajardo, M., Olguin-Araneda, V., Barra-Carrasco, J., Brito-Silva, C., Sarker, M. R., \& Paredes-Sabja, D. (2014). Characterization of the collagen-like exosporium protein, BclA1, of Clostridium difficile spores. Anaerobe, 25, 18-30. doi:10.1016/j.anaerobe.2013.11.003.

45. Janganan, T. K., Mullin, N., Tzokov, S. B., Stringer, S., Fagan, R. P., Hobbs, J. K., . . B Bullough, P. A. (2016). Characterization of the spore surface and exosporium proteins of Clostridium sporogenes; implications for Clostridium botulinum group I strains. Food Microbiol, 59, 205-212. doi:10.1016/j. fm.2016.06.003.

46. Roberts, T. A. (1968). Heat and radiation resistance and activation of spores of Clostridium welchii. Journal of Applied Bacteriology, 31(1), 133-144.

47. McDonnell, G., \& Russell, A. D. (1999). Antiseptics and disinfectants: activity, action, and resistance. Clinical Microbiology Reviews, 12(1), 147-179.

48. Barbut, F., Menuet, D., Verachten, M., \& Girou, E. (2009). Comparison of the efficacy of a hydrogen peroxide dry-mist disinfection system and sodium hypochlorite solution for eradication of Clostridium difficile spores. Infection Control and Hospital Epidemiology, 30(6), 507-514. doi:10.1086/597232.

49. Perez, J., Springthorpe, V. S., \& Sattar, S. A. (2005). Activity of selected oxidizing microbicides against the spores of Clostridium difficile: relevance to environmental control. American Journal of Infection Control, 33(6), 320-325. doi:10.1016/j.ajic.2005.04.240.

50. Evaluation Procedure for Gray Scale for Color Change, EP12018 (2020). In: AATCC Technical Manual, Association of Textile Chemists and Colorists, Research Triangle Park, NC.

51. Instrumental Color Measurement, EP6-2016 (2017), In: AATCC Technical Manual, Association of Textile Chemists and Colorists, pp. 430-436, Research Triangle Park, NC.

52. Guan, J., Chan, M., Brooks, B. W., \& Rohonczy, L. (2013). Influence of temperature and organic load on chemical disinfection of Geobacillus steareothermophilus spores, a surrogate for Bacillus anthracis. Canadian Journal of Veterinary Research, 77(2), 100-104.

53. Cadnum, J. L., Mana, T. S., Jencson, A., Thota, P., Kundrapu, S., \& Donskey, C. J. (2015). Effectiveness of a hydrogen peroxide spray for decontamination of soft surfaces in hospitals. American Journal of Infection Control, 43(12), 1357-1359. doi:10.1016/j.ajic.2015.07.016. 